\title{
Rutas contra el silencio: análisis de los mecanismos para el manejo y prevención del acoso sexual al interior de la Universidad de Antioquia (Colombia)
}

\author{
Routes against silence: An analysis of the mechanisms for the management \\ and prevention of sexual harassment within the University of Antioquia \\ (Colombia)
}

\author{
Por: Andrea Olaya Martínez \\ 1. Politóloga de la Universidad de Antioquia. Integrante de la Red Nacional Universitaria por la equidad de género en la educación superior. \\ Contacto: andrea.olaya@udea.edu.co Orcid: https://orcid.org/0000-0002-2729-6687 Orcid: https://orcid.org/0000-0002-2729-6687
}

\section{OPEN ACCESS}

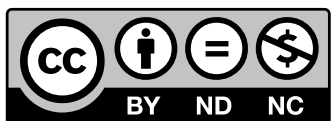

Copyright: ( 2020 El Ágora USB.

La Revista El Ágora USB proporciona acceso abierto a todos sus contenidos bajo los términos de la licencia creative commons AtribuciónNoComercial-SinDerivar 4.0 Internacional (CC BY-NC-ND 4.0)

Tipo de artículo: Investigación

Recibido: mayo de 2019

Revisado: agosto de 2019

Aceptado: diciembre de 2019

Doi: $10.21500 / 16578031.4137$

Citar así:Olaya Martínez, A. (2020). Rutas contra el silencio: análisis de los mecanismos para el manejo de acoso sexual al interior de la Universidad de Antioquia (Colombia). El Ágora USB, 20(1). 142-156. DOI:

$10.21500 / 16578031.4137$

\section{Resumen}

En un momento histórico para el feminismo en el que la conversación sobre el acoso sexual toma fuerza, este trabajo decide indagar sobre el papel de las universidades como instituciones que deben garantizar la seguridad, integridad y salud de sus estudiantes; específicamente la Universidad de Antioquia. El texto se pregunta por la existencia de un protocolo que apoye a los estudiantes víctimas de acoso sexual en la universidad y utiliza una metodología que está dividida en tres etapas: la primera etapa hace una exploración de conceptos académicos y jurídicos que provean una guía acerca de los protocolos que deberían encontrarse; en la segunda etapa se analizan los hallazgos de la primera etapa de una forma crítica, en la tercera etapa se realiza la construcción y sugerencia de un protocolo que se ajuste al contexto de la universidad de Antioquia. Finalmente, se presentan una serie de conclusiones en torno a dichos protocolos y la importancia de una política formal institucional que prevenga, sancione y sensibilice en el tema.

Palabras clave. Acoso sexual universitario; violencia sexual en la universidad; estudiantes, universidades.

\section{Abstract}

At a historic moment for feminism in which conversation about sexual harassment takes hold, this work decides to inquire about the role of universities as institutions that must ensure the safety, integrity, and health of their students; specifically the University of Antioquia. This text asks about the existence of a protocol, which supports students who are victims of sexual harassment at the university, and it uses a methodology, which is divided into three stages: the first stage explores academic and legal concepts that provide a guidance on the protocols that should be found. In the second stage, the findings of the first stage are analyzed in a critical way, and in the third stage, the construction and suggestion of a protocol that conforms to the context of the University of Antioquia is carried out. Finally, a number of conclusions are presented around these protocols and the importance of a formal institutional policy that prevents, sanctions, and sensitizes on the issue.

Keyword. University's Sexual Harassment; Sexual Violence at University; Students; and Universities. 


\section{Introducción}

Este artículo surge como respuesta a experimentar de primera mano el acoso sexual durante la práctica profesional y las repercusiones que este tipo de conductas sobre la víctima. Esta problemática que hoy ha sido llevada a la agenda pública por movimientos como el \#MeToo y el \#BalanceTonPorc, movimiento feminista nacido en Estados Unidos que aboga por acabar con años de silencio frente al acoso sexual denunciando púbicamente a los acosadores. Nace en Twitter con el hashtag popularizado por la actriz Alyssa Milano. Al igual que el \#BalanceTonPorc nacido en Francia, ha puesto de manifiesto que este tipo de conducta lleva años extendiendo sus raíces y sosteniendo jerarquías que profundizan las asimetrías de poder en las relaciones de género, estando presente en diferentes espacios como las empresas, las familias e incluso las universidades.

La relevancia que esta problemática ha ganado, ha servido para evidenciar que la sociedad carece de experiencia para afrontarla y que la cultura del silencio que había imperado hasta hace poco sirvió para proteger a los acosadorxs, perpetuando la impunidad y alentando la normalización de dichos comportamientos. De ello da cuenta la experiencia vivida, en la que, ante la ausencia de protocolos o mecanismos formales para tramitar la denuncia, se terminó optando por desistir de iniciar un proceso formal al interior de la institución educativa que permitiera dar una solución a la situación y evitara la revictimización.

La tarea de responder al acoso sexual es entonces hoy en día tan desalentadora como urgente. En la mayoría de los casos de acoso sexual, los victimarixs han enfrentado las mismas consecuencias: ninguna. Tres de cada cuatro casos de acoso sexual no son reportados por las víctimas por miedo a represalias, ya que la autoridad y poder del victimarix, o incluso el mismo sistema Ixs ayuda a mantener su reputación y carreras intactas (Aguirre Fernandez, 2017).

Ejemplo de la dificultad de afrontar el acoso sexual es que la respuesta al malestar social generado por el sinnúmero de denuncias en muchos casos haya terminado afectando a las víctimas, que ven sus carreras profesionales afectadas por situaciones que incluyen hombres inversionistas rehusándose a reuniones a solas con mujeres, moverlas de un restaurante a una sala de conferencias, que los hombres eviten reuniones a puerta cerrada con ejecutivas junior, entre otras similares Miller, C. (2017).

Este panorama demuestra la necesidad de evitar que situaciones como la vivida se normalicen y hagan parte de la cotidianidad de las víctimas, generando afectaciones en sus ámbitos académicos y laborales, o incluso en su desenvolvimiento en tales espacios, pues en muchas ocasiones las respuestas de las víctimas van desde el aislamiento y evitación de los espacios frecuentados por el agresor, a cuadros de angustia, estrés y sensación de minusvalía.

Esta es una labor que requiere de compromiso institucional. Es necesario contar con mecanismos formales y protocolos que aseguren el acompañamiento, trazo claro de rutas a activar en dichas situaciones, conductos regulares que brinden seguridad, tranquilidad y discreción, que garanticen que la denuncia no va a transformarse en revictimización y la oportunidad de represalias por parte del victimarix.

Por ello este trabajo indaga sobre la existencia de mecanismos o protocolos dentro de la Universidad de Antioquia para hacer frente al acoso sexual, tratándose de la institución en cuyo programa de prácticas fue vivida la experiencia de acoso sexual, y de existir dichos mecanismos, se evalúa su idoneidad al compararlos con protocolos y estándares internacionales. Se trata de un caso relevante pues es una de las universidades más destacadas del país en temas de investigación (Sapiens Research, 2019) y el claustro con el mayor número de estudiantes en la ciudad de Medellín (Universidad de Antioquia, 2019). 
Esta indagación tendrá tres momentos. Un primer apartado recogerá diferentes definiciones que se han dado del acoso sexual y sus diferencias con el abuso sexual, para ofrecer al lector una definición sobre la cuál pueda guiarse el análisis de los protocolos. Tras una breve consideración metodológica, se presentarán entonces los hallazgos sobre mecanismos al interior de la Universidad, luego se agrupan diferentes protocolos cuya efectividad o idoneidad ha sido reconocida por instituciones dedicadas a trabajar el tema del acoso sexual, resaltando los elementos comunes que debería tener en cuenta todo diseño de instrumentos dirigidos a atacar esta problemática para finalmente, producto de la comparación, elaborar una serie de recomendaciones sobre las consideraciones y principios necesarios para asegurar la idoneidad de cualquier herramienta que se busque implementar para el manejo y prevención del acoso sexual al interior de la Universidad de Antioquia.

\section{Hacia una definición amplia del acoso sexual}

Al abordar el concepto de acoso sexual, pueden identificarse tres grandes momentos en su desarrollo. Un primer momento en que aparece como denuncia de diferentes colectivos con ánimo reivindicatorio; seguido por la represión judicializada, en la que los gobiernos buscaron tipificar y sancionar este tipo de conductas como delito; y finalmente, su abordaje desde los estudios académicos, que buscan delimitar el concepto, definiendo cuáles conductas constituyen acoso y cuáles no (Caballero, 2003).

Reconociendo este recorrido, es necesario partir para la delimitación del concepto de acoso sexual desde la definición que el legislador colombiano hizo de esta conducta, contenida en el artículo 210A del Código Penal que lo define como

El que en beneficio suyo o de un tercero y valiéndose de su superioridad manifiesta o relaciones de autoridad o de poder edad, sexo, posición laboral, social, familiar o económica, acose, persiga, hostigue o asedie física o verbalmente, con fines sexuales no consentidos, a otra persona.

En un posterior desarrollo del concepto en la jurisprudencia de la Sala de Casación Penal de la Corte Suprema de Justicia en sentencia SP107-2018, se diferenció además el acoso sexual de otros delitos sexuales, estableciendo que,

(...) si bien, no se posee una definición unívoca de acoso sexual, sí es posible determinar un lugar común, referido a que se trata de actitudes o comportamientos que por sí mismos causan mortificación o crean un clima hostil en ámbitos de trabajo o similares, respecto de actos, gestos o palabras que en muchas ocasiones representan una pretensión, pero no la consumación de la misma.

Igualmente, es relevante revisar el desarrollo que se ha hecho del concepto de acoso sexual en el derecho internacional, en el que uno de los principales referentes es la definición dada por la Organización Internacional del Trabajo (OIT), que la tipifica como un "[c] omportamiento en función del sexo, de carácter desagradable y ofensivo para la persona que lo sufre. Para que se trate de acoso sexual es necesaria la confluencia de ambos aspectos negativos: no deseado y ofensivo" (2012, p.1).

Una conceptualización más extensa en el derecho internacional es la ofrecida por la Convención para la eliminación de todas las formas de discriminación contra las Mujeres (CEDAW por sus siglas en inglés), que la define como:

Comportamiento de tono sexual tal como contactos físicos e insinuaciones, observaciones de tipo sexual, exhibición de pornografía y exigencias sexuales, verbales o de hecho. Este tipo de conducta 
puede ser humillante y puede constituir un problema de salud y de seguridad; es discriminatoria cuando la mujer tiene motivos suficientes para creer que su negativa podría causarle problemas en el trabajo, en la contratación o el ascenso inclusive, o cuando crea un medio de trabajo hostil.

En una línea similar a la OIT y a la CEDAW, organizaciones especializadas se limitaron a definir el acoso sexual como una conducta específica del ámbito laboral y educativo. Dos ejemplos son las definiciones ofrecidas por la Comisión para la Igualdad de Oportunidades en el Empleo de los Estados Unidos (EEOC por sus siglas en inglés), y por la Oficina para los Derecho Civiles del Departamento de Educación de los Estados Unidos (OCR por sus siglas en inglés), quienes la definen como una conducta no correspondida de naturaleza sexual que limita u obstaculiza el desempeño de la víctima en los ámbitos educativo o laboral Education Department, (2001).

Ya a nivel académico algunos de los primeros intentos por definir el acoso sexual partieron de las definiciones dadas por el legislador, para buscar ampliarlas o enriquecerlas. En esta línea se inscribe el trabajo de Maria C. Caballero, que partiendo de la definición del Código Penal Español, que denomina acoso sexual formal, y en el que el énfasis está puesto en la coerción,

El que solicitare favores de naturaleza sexual, para sí o para un tercero, en el ámbito de una relación laboral, docente o de prestación de servicios, continuada o habitual, y con tal comportamiento provocare a la víctima una situación objetiva y gravemente intimidatoria, hostil o humillante, será castigado, como autor de acoso sexual, con la pena de prisión de tres a cinco meses o multa de seis a 10 meses. (...). (Art. 184).

desarrolla la idea de que las que denomina conductas de incomodidad, "como el uso del lenguaje obsceno, la falta de respeto, las bromas sobre el sexo, realizar actividades domésticas no contempladas en las funciones y permitir el consumo de bebidas alcohólicas en los lugares de trabajo y estudio" (Caballero, 2003, p.441) son un indicador de casos de acoso sexual formal, pues su presencia aumenta las posibilidades de su ocurrencia.

Otra aproximación fue la de intentar identificar las conductas o acciones que se constituían como acoso sexual. Este tipo de trabajos sugieren que el acoso puede adoptar diversas formas, desde la coerción física hasta el uso del poder mediante el ofrecimiento de recompensas, prebendas, o la negación de derechos adquiridos; modalidades que van desde actos sexistas y degradantes, comunes en el cotidiano, seguido por avances sexuales no deseados, chantaje y coerción, hasta asaltos o ataques físicos con fines sexuales. Igualmente, según este tipo de trabajos existe confusión entre seducción y hostigamiento, entre un intercambio amoroso consentido y una conducta de naturaleza sexual, sorpresiva, que no es recibida con agrado (Castaño Castrillón et al, 2008).

En las definiciones más recientes, el énfasis está puesto ya en el carácter violento de la conducta y su relación con la discriminación de género, así la Corporación Sisma Mujer describe el acoso como

(...) una de las formas de violencia sexual que se comenten contra miles de mujeres en el mundo y se encuentra naturalizada en las relaciones sociales y laborales, al punto de considerar que es una vivencia común en la vida laboral de las mujeres, que no es objeto de sanciones -sociales, penales ni disciplinarias $(2013$, p.17).

Sin embargo, estas definiciones del acoso sexual que lo definen como una conducta, y limitan su ocurrencia a los entornos laboral y académico, terminan por ocultar las 
condiciones sociales que han permitido y normalizado su ocurrencia en un sinnúmero de interacciones que ocurren desde el hogar hasta el espacio público. Además, el excesivo énfasis puesto en el género y condición de inferioridad de la víctima, invisibiliza la ocurrencia de casos de acoso entre personas del mismo estatus, género y condición social (Gaytán, 2009).

Por ello se opta en este trabajo por una definición amplía que presente el acoso no como una conducta, concentrándose en el perfil sicológico del acosador, o las condiciones dentro de espacios determinados que incentivan el acoso, sino una que preste atención a las condiciones sociales generales que posibilitan el acoso como un cierto tipo de interacción entre dos o más personas. Se entenderá entonces por acoso sexual,

(...) una o varias interacciones focalizadas cuyos marcos y significados tienen un contenido alusivo a la sexualidad, en las que la actuación de al menos uno de los participantes puede consistir en aproximaciones sexuales indirectas (empleo de símbolos, mensajes escritos, silbidos a distancia, material pornográfico), soborno sexual, acercamientos, miradas, susurros y contactos físicos o proposiciones y comentarios sexuales que no son autorizados ni correspondidos, generan un entorno social hostil y tienen consecuencias negativas para quien las recibe. Es posible que involucren diferencias de jerarquía y estatus, y necesariamente implican un desequilibrio en las relaciones de poder entre los individuos que puede ser contrarrestado o no durante la misma situación. Ocurre en diferentes medios (Gaytán, 2009, p.53).

\section{Metodología}

Para responder a la interrogante sobre la existencia o no de mecanismos para afrontar y prevenir el acoso sexual al interior de la Universidad de Antioquia, se presenta al lector una investigación documental, esta es una metodología de investigación que por medio de la revisión de documentos de diferente tipo logra dar cuenta de un problema social. La técnica de análisis es el análisis crítico que consiste en la interpretación del significado de los documentos en su contexto de elaboración para establecer recurrencias Valles, M. (1999).

Para el análisis crítico de los documentos se usaron siete categorías extraídas de una serie de recomendaciones hechas por OCR (2001) y la investigación de Kimberly Cummings y Madeleine Armenta (2002), que permiten establecer la idoneidad de un protocolo, siendo las categorías: 1. Conductas sancionadas o prohibidas (¿Delimita el concepto de acoso sexual? ¿Es una definición amplia que permita sancionar las formas sutiles de acoso sexual?); 2. Principios rectores (¿Garantiza la reparación, garantía de no repetición y acceso a la verdad, y evita la revictimización?); 3. Alcance (¿Se trata de un protocolo general o existen diferentes protocolos para cada dependencia?); 4. Actores (¿Se establece una ruta que propicie la cooperación de las diferentes áreas implicadas?); 5. normativa aplicable (¿El protocolo es incluido en los reglamentos de la institución? ¿Está sintonizado con la normativa nacional e internacional?); 6. tipo de sanción (¿La sanción es pedagógica, laboral, disciplinaria?); y 7. Canales de comunicación (¿Los canales para la denuncia protegen la identidad de la víctima? ¿Se evita que la víctima deba volver varias veces a narrar el suceso traumático?).

Para reunir la documentación existente al interior de la Universidad de Antioquia sobre rutas o protocolos para el manejo del acoso sexual se hizo una consulta al sistema de consulta de documentos digitalizados sobre Normativa Universidad de Antioquia y se enviaron tres derechos de petición a la Secretaría General, al Consejo de Facultad de Derecho y Ciencias políticas, y a Bienestar Universitario. En cuanto a los protocolos externos, se recogieron un total de ocho documentos, teniendo como criterio de selección el que su 
efectividad o idoneidad haya sido reconocida por instituciones gubernamentales u organizaciones dedicadas a combatir el acoso sexual.

Finalmente, se compararon los resultados del análisis de los diferentes protocolos externos, con los de los mecanismos al interior de la Universidad de Antioquia, para establecer las diferentes consideraciones que deben incluirse en los instrumentos que se busque implementar para el manejo y prevención del acoso sexual al interior del claustro.

\section{Del manejo del acoso sexual en la Universidad de Antioquia}

El primer acercamiento a la normativa y regulación formal al interior de la universidad se efectuó a través de la plataforma "Normativa Universidad de Antioquia; sistema de consulta de documentos digitalizados" (Universidad de Antioquia, 2018). Dicha plataforma, constituye la base de datos en la que la universidad consigna las resoluciones, acuerdos, actas, comunicados y circulares del Consejo Superior Universitario, Consejo Académico y Secretaría General, las resoluciones rectorales y reglamentos que rigen estudiantes, docentes y personal administrativo. Dicha búsqueda no arrojó ningún resultado; no fue posible rastrear un protocolo o incluso la mención específica de las palabras acoso sexual.

Paralelamente, fueron enviados tres derechos de petición a tres dependencias de la universidad; el primero, dirigido al Consejo de Facultad de la Facultad de Derecho y Ciencia política, el segundo, a la Dirección General de Bienestar universitario y el tercero, a la Dirección Jurídica de la Universidad. Considerando que son las instancias formales encargadas del cumplimiento de los diferentes reglamentos consultados (Bienestar Universitario, Disposición rectoral de regulación de acoso laboral, estatuto profesoral con concordancias y el reglamento estudiantil con concordancias) y los programas que se desarrollan tanto en las facultades como en la dirección General de Bienestar, que incluyen programas de salud sexual y reproductiva, se solicitó información precisa, sobre la existencia, construcción, o precedentes formales de atención a denuncias de acoso sexual por parte de los estudiantes:

Asunto: Solicitud de documentación, indicaciones, claridades sobre las rutas y conductos regulares establecidos a seguir en el eventual caso de una denuncia de acoso sexual al interior de la facultad y sus centros de práctica. (...) que me sean indicadas las reglamentaciones, rutas, manejo, personas a cargo, conductos regulares, escalamientos y procedimientos que se han seguido o se deben seguir en el caso de una denuncia por acoso sexual al interior de la Universidad en sus facultades y en sus centros de práctica. Que me sea indicado qué manejo se le da institucionalmente a este tipo de eventualidades. Y, en caso de que no exista dicha ruta para el caso específico de acoso sexual, me sea notificado por escrito.

La Dirección General de Bienestar Universitario no respondió al derecho de petición.

El Consejo de facultad de Derecho y Ciencia Política, respondió el día 16 de abril del presente año, indicando que:

Existe actualmente en la Facultad el Comité de Asuntos de Género. (...) Desde el CAF se está construyendo de manera interdisciplinaria un documento con una ruta para los casos o situaciones relacionadas con acoso sexual, a la fecha se encuentran levantando información documental sobre qué se hace en otras universidades e instituciones nacionales e internacionales sobre el tema, se ha hecho un rastreo bibliográfico para construir un concepto de acoso sexual y se ha avanzado en la revisión de asuntos legales, jurisprudenciales y de Derecho Comparado con la legislación en 
otros países; asimismo, se ha solicitado a la Fiscalía capacitación relacionada con los procesos de acoso sexual y "material estadístico sobre denuncias particularmente de población universitaria"

En cuanto al manejo dado a los casos hasta ahora por el Consejo de Facultad se dijo que, "(...) cuando los casos son conocidos por la Coordinación de Bienestar en la Facultad, son remitidos a Promoción de la salud y Prevención de la Universidad (...)", y se expresó que.

(...) la ruta se encuentra en construcción y, por tanto, si bien hay profesores y programas en la Facultad ocupándose del tema, no hay actualmente una reglamentación en la que se establezcan los procedimientos a seguir "(...) en el caso de una denuncia por acoso sexual al interior de la Facultad" y tampoco se cuenta a la fecha con una ruta establecida.

A la fecha de entrega de este trabajo, el documento sigue sin ser entregado. Por su parte, la Dirección Jurídica de la Universidad respondió:

(...) una de las normas regulatorias de los eventos del acoso sexual, es la ley 1010 del 2006, toda vez que éste es una de las modalidades de acoso laboral. Asimismo, no sobra anotar que el acoso sexual también está tipificado en el artículo 210 A del Código Penal (Ley 599 de 2000), adicionado por el artículo 29 de la ley 1257 de 2008 (...)

\section{Y continúa:}

Es de resaltar, que las situaciones de acoso laboral conocidas por el comité de Convivencia Laboral, son las que ocurren en el ámbito de relaciones de dependencia o subordinación de carácter laboral. Lo anterior significa, que los presuntos casos de acoso sexual denunciados por los estudiantes de la institución, en contra de profesores y empleados administrativos no serán conocidos por dicho Comité y, por el contrario, serán competencia de la Unidad de Asuntos Disciplinarios de la Universidad de conformidad con los Acuerdos Superiores 262 de 2003 y 297 de 2005.

Al analizar la información expuesta por la Dirección jurídica, tras soportar que efectivamente no existe un protocolo que maneje específicamente las denuncias de acoso sexual, sino por vía de relación laboral, que sólo cobija empleados y la remisión a los dos acuerdos superiores previamente mencionados, se encontró que en el Acuerdo 262 de 2003 (Universidad de Antioquia, 2006) no se encuentra nada en relación con el acoso sexual, y en el Acuerdo 297 de 2005 por el cual se establece el régimen disciplinario del Personal Docente de la Universidad de Antioquia (Universidad de Antioquia, 2005), se encontró que en el Artículo 14, letra g, se constituye una falta gravísima "Violentar, exigir o constreñir a un miembro de la comunidad universitaria, manifestando inequívocamente una pretensión sexual, de manera insistente y no querida por éste." Si bien éste único hallazgo significa un punto indiscutible de partida, no constituye un mecanismo o una herramienta útil para el manejo de las denuncias de acoso sexual al interior de la Universidad.

Por ende, puede afirmarse que en la Universidad de Antioquia no existen rutas o protocolos formales para el manejo de las denuncias por acoso sexual que se presenten al interior de la universidad y en sus centros de extensión y prácticas por parte de Ixs estudiantes. Lo cual dificulta, como en el caso vivido, que haya incentivos para denunciar. 


\section{Otras aproximaciones al manejo del acoso sexual en las instituciones}

Con el objetivo de proponer un protocolo amplio, eficiente y accesible para lo estudiantes, se responde a los principios de verdad, reparación y garantía de no repetición.

Como ya ha sido mencionado es indispensable que se cuente con una definición amplia y clara de qué conductas constituyen acoso lo cual debería facilitar a las víctimas reconocerse y decidir si desea activar o no el protocolo.

Algunas de las conductas que constituyen acoso sexual son:

- Bromas sexuales, o insinuaciones sexuales repetidas en persona o por correo.

- Abuso verbal de naturaleza sexual.

- Tocar o agarrar de manera sexual.

- Pararse muy cerca o tocarse "accidentalmente" con una persona de manera repetitiva.

- Pedirle repetitivamente a una persona que socialice fuera del trabajo cuando la persona ha dicho no o ha indicado que el o ella no está interesado(a) (los supervisores en particular deben ser muy cuidadosos de no presionar a sus empleados de socializar).

- Dar regalos o dejar objetos que son sexualmente sugestivos.

- Hacer expresiones sexualmente sugestivas en repetidas ocasiones.

- Hacer o colocar imágenes, caricaturas o algún otro material sexualmente ofensivo o degradante en el lugar de trabajo.

- Comportamientos molestos de naturaleza sexual fuera del trabajo, que afectan el ambiente laboral.

En 2012, mediante un estudio de encuestas la Corporación Sisma Mujer (2013, p.11) estableció que el 85\% de las mujeres colombianas reconoció el acoso sexual en el trabajo como una problemática vigente. La sensación de riesgo es mayor en Barranquilla (89\%), Cali (89\%), Bogotá (85\%) y Medellín (78\%).

En estudios reportados en la Universidad de Manizales se plantea que los estudiantes parecen responder enérgicamente a favor de las víctimas que informan sobre los incidentes del acoso sexual.

Por lo anterior, en lugar de concentrarse en políticas de entrenamiento de cuerpo docente, personal administrativo, y asesoramiento de víctimas, los esfuerzos deben ser encausados para un cambio de ambiente en el campus, de manera que sea hostil hacia el acoso sexual. En cuanto al castigo apropiado que debería recibir el acosador para su penalización, en el caso colombiano se han expedido múltiples normas, entre las que se cuentan la ley 248 de 1995 15, las reformas al Código Penal de la ley 599 del 200016, la Ley 294 de 1996 o de violencia intrafamiliar, Ley 1010 de enero de 200618, que castigan el acoso laboral, y la Ley de la Infancia y la Adolescencia de noviembre de 2006 (Castrillón et. al, 2008: 4).

De igual forma, se sugiere que el protocolo sea seguro, discreto, que proteja la identidad de denunciante y denunciado, lo cual es indispensable para evitar la revictimización, y el miedo al escarnio público tras la denuncia.

Respecto al alcance: Se recomienda un protocolo general, centralizado, lo cual no sólo facilita su manejo, sino que garantiza orden, sistematización y habilidad en la recepción y trámite de las denuncias.

Sobre la jurisdicción: 
- Estudiantes: Cuando la queja sea contra un estudiante, el oficial dará manejo a la misma en nombre de la universidad.

- Miembros de la Facultad (Docentes o directivos): La jurisdicción de las quejas contra miembros de una facultad dependerá de las circunstancias de la queja, las cuales serán determinadas por los siguientes factores:

- A. ¿Qué institución tiene responsabilidad inmediata sobre la persona denunciada? B. ¿Dicha institución posee la capacidad de regular la conducta de la persona denunciada, imponer sanciones o restricciones de acceso a las instalaciones de la universidad o a la persona que denuncia?

- C. ¿Es dicha institución la principal responsable de proteger los intereses de la persona denunciada de un proceso justo en imparcial?

- D. ¿Es dicha institución la principal responsable de salvaguardar el ambiente laboral y académico de la persona que denuncia?

- Otras partes involucradas: En las circunstancias en las que sea la Universidad (oficial designado) sea quien se haga cargo de la denuncia, independiente de qué institución sea la instancia primaria de atención, ambas deberán interesarse y enterarse del manejo y resolución de dicha denuncia. Una vez tomada una decisión, la institución que no posee jurisdicción será invitada a reiterar y apoyar la decisión y resolución en la denuncia.

- "Procedimientos y jurisdicción de la Universidad de Toronto: cuando las denuncias sean tramitadas ante la oficina de acoso sexual, el oficial deberá invitar al denunciado y a su superior inmediato a manifestar su posición frente a la denuncia." (Universidad de Toronto. 2004: 6).

Respecto a los actores involucrados, se requiere de la cooperación y trabajo conjunto entre la persona designada como el/la oficial del protocolo y el personal a cargo de la dependencia a la que el estudiante pertenezca. (Yale, 2018, p.7).

Finalmente, es necesario contemplar el tipo de sanciones que se contemplarán en dicho protocolo, para lo cual, considerando acciones amplias dentro de lo que se limita a la reglamentación universitaria, distinto a lo que pueda contemplar la justici ordinaria (En caso de que haya necesidad de acudir a ella) nos referimos a lo propuesto en el estudio de las autoras Cummings y Madeline (2002: 274) quienes contemplan seis posibles sanciones para quien acose: 1. Una sanción no disciplinaria, en la que la víctima recibe una disculpa por escrito por parte de su acosador. 2. El/La acosador(a) recibe un llamado de atención por escrito (Incluido en su hoja de vida). 3. El/La acosador(a) accede a recibir formación en temas de género y acoso sexual. 4. El/La acosador(a) es suspendidx temporalmente de su cargo en la Universidad. 5. El/La acosador(a) es expulsado de manera permanente de la universidad. 6. Se da trámite externo ante las autoridades competentes.

\section{Recomendaciones para las víctimas}

Tanto hombres como mujeres son objeto de acoso sexual, si bien los estudios ponen de relieve que la mayoría son mujeres. La investigación muestra que el tipo de mujer más vulnerable al acoso sexual es la mujer joven, económicamente dependiente, soltera o divorciada y las que tienen estatus de inmigrante. Con respecto a los hombres, aquellos que sufren un mayor acoso son los jóvenes, homosexuales y miembros de minorías étnicas o raciales. No obstante vale la pena resaltar que el acoso afecta a mujeres en todos los niveles jerárquicos y tipos de trabajo (Organización Internacional del Trabajo, 2012).

El acoso casi diario infunde temor a la víctima, lo que conlleva a que se mantenga aislada de espacios donde el agresor pueda encontrarse. Las repercusiones pueden ser psíquicas (reacciones relacionadas con el estrés como traumas 
emocionales (ansiedad, depresión, estados de nerviosismo, sentimientos de baja autoestima), y físicas (trastornos del sueño, dolores de cabeza, problemas gastrointestinales, hipertensión) (Organización Internacional del Trabajo, 2012).

La dificultad más frecuente para las víctimas es el temor a denunciar por las represalias de sus superiores. Caballero (2003) se refiere a las tres etapas del proceso histórico por el que ha pasado el acoso (La denuncia, la represión judicializada y el estudio académico), y advierte que en muchos países como el nuestro, en los que las conductas de acoso sexual han sido naturalizadas y donde se responsabiliza a las víctimas por sus efectos negativos, es posible que falte desarrollar o completar esta etapa de denuncia. "La respuesta del 60\% de las mujeres víctimas de acoso sexual formal es la huida y solamente un $30 \%$ de las mujeres se enfrenta al agresor. La respuesta de huida la podemos explicar si consideramos el grave riesgo a que se expone la mujer en el caso de una confrontación con el agresor" (Caballero, 2003: 445).

Por ello se establecen unas indicaciones necesarias para que una víctima de acoso tenga herramientas para combatirlo

- Lo primero es tener claro qué es el acoso sexual.

- Acudir en búsqueda de atención médica si fuiste heridx o asaltadx sexualmente.

- Es importante reconocer que no estxs solx, muchas personas han experimentado el acoso sexual, y ni ellxs ni tú, son culpables.

- Cuéntale a alguien de tu confianza lo sucedido, esto te ayudará a comenzar a sanar y avanzar en la reparación y decisión de acción u omisión.

- Informarse de los mecanismos a tu disposición para denunciar, ya sea por vía legal, o ante la institución a la que pertenezca la persona que te abusó.

- Es bueno detenerse a pensar sobre cómo te sientes. Tras palabras o actos en los que sufres acoso sexual, puedes sentirte en shock, confundida, avergonzada, culpable, enojada, ansiosa o no sentir nada en absoluto. Estas son reacciones perfectamente normales; es importante que las reconozcas ya que esto determinará qué tipo de ayuda buscarás a continuación.

- Es importante que localices y de ser necesario te dirijas a personas o instituciones que puedan brindarte asesoría como organizaciones feministas, refugios de mujeres, secretarías de la mujer, líneas telefónicas de atención psicológica y de crisis (Como la línea Amiga en Medellín).

- Si te sientes asustada, es importante que busques un lugar seguro dónde quedarte por al menos un par de días, un familiar o una amiga empática son una buena opción.

\section{La construcción de Protocolos}

La Constitución de 1991, la ratificación de la CEDAW, la ley 248 del 95, ONU 2017 y los principios de Yogyakarta, la declaración de orientación e identidad de género de la ONU, y la ley 1257 del 2008 son algunas de las disposiciones que fundamentan y regulan el tema y en las que se pueden enmarcar los protocolos institucionales.

Es necesario que se haga una definición clara y amplia sobre qué es el acoso sexual (Enseñar ejemplos claros sobre qué conductas constituyen acoso sexual) y que a su vez el protocolo delimite quiénes y qué conductas, deben regirse por él.

En el aspecto de la capacitación, es necesario que toda cláusula de manejo interno y confidencialidad en denuncias de índole sexual sean removidas de los contratos; por el contrario, pueden agregarse "otro sí" a los ya realizados en los que tras la capacitación recibida, cada empleado o estudiante firme haber entendido los protocolos, rutas y prohibiciones expresas sobre el acoso sexual en la institución. 
El Departamento de Recursos humanos de la institución debe ser notificado y estar atento al resultado cada que haya una investigación en curso.

Durante y después de la investigación, es responsabilidad de la institución garantizar que la víctima no sufra retaliación por parte de la persona que a la que denunció y que reciba atención de consejería o psicología por parte de la institución.

Este protocolo cobija todos los integrantes que hagan parte del campus universitario como estudiantes, practicantes, docentes, personas en la nómina de la universidad, miembros del sistema de salud de la Universidad y personal administrativo.

El protocolo que se sugiere, contempla que si bien es posible que cada Facultad, Escuela, División Administrativa puede o no tener su propio protocolo, el general acá propuesto no modifica ni reemplaza ninguno de esos, y que deben desarrollarse bajo los parámetros del general.

Lo primero que se sugiere es la notificación de la denuncia; la cual debe ser específica en el lugar de los hechos y las personas implicadas para que la Universidad pueda corroborar que se trata de miembros de la misma y que los hechos se dieron delimitados en la jurisdicción de la universidad. Dicha queja debe ser entregada cuanto antes sea posible tras los hechos al "Oficial De Acoso Sexual" de la Universidad el o la cual deberá hacer las dos siguientes preguntas:

- ¿Qué institución(es) posee autoridad, capacidad de sanción y responsabilidad de supervisión y administración de la persona acusada de acoso sexual?

- ¿Qué institución(es) poseen obligaciones o compromisos (como la garantía de los derechos) de la persona que está denunciando haber sidx acosadx?

Personas que deben ser notificadas:

El oficial deberá notificar a cada una de las instituciones mencionadas en la respuesta a las dos anteriores preguntas, deberá ser específico en la naturaleza de la denuncia, y exigir que se interesen por dicha denuncia.

Posteriormente si la persona denunciada es un empleado, recursos humanos debe ser notificado de la denuncia para estar atentos a la resolución que se dé al caso tras ser investigado.

Como mecanismo de pedagogía y prevención, cada estudiante, docente y empleado debe ser informado de los procedimientos de denuncia basándose en la necesidad de conocerlos y su obligación de actuar. Cada que el oficial designado para el manejo de acoso sexual sea indagado por la ruta deberá proveer la información clara para que la persona que consulta tenga las herramientas para denunciar y tomar decisiones sobre la jurisdicción.

Debe consignarse en el protocolo a construir si la víctima no acude a las autoridades, la institución lo haga con su representante, no obstante se considera que para evitar la revictimización, la instancia jurídica debería ser una decisión exclusiva de la víctima.

A su vez hay protocolos como el de la Iglesia Anglicana de Australia (2015) que contemplan la posibilidad de que la denuncia sea interpuesta por un tercero, aspecto que podría contemplarse siempre y cuando la víctima sea quien decida si desea continuar con dicho proceso. Es importante "empoderar" a los espectadores para que consideren confrontar al acosador, o a manifestar su apoyo a la víctima: ofreciéndose como testigo en la denuncia, interrumpiendo o "rescatándola de una situación incómoda, recordándole que no es su culpa.

De igual forma, como lo contempla el protocolo de la ESADE "Protocol for prevention and action in situations of mobbing and sexual harassment" (2017, p.4), se procura que no haya una necesidad por parte de la víctima de narrar los hechos una y otra vez, sino que, a su disposición estén los medios para que su testimonio se tome por escrito, visual 
o en audio. De esta manera se evita también una posible re-victimización al reiterar en lo que pueda sentir al narrar los hechos. Dicho testimonio debe ser lo más detallado posible.

En el caso del protocolo construido de forma minuciosa por la Iglesia Anglicana de Brisbane (2015), hay un detalle que debe ser considerado, y es el hecho de que incluso siendo mayor de edad, en el caso de que la víctima sea una persona con cualquier tipo de discapacidad, debe estar siempre acompañada por un guardián (representante familiar, o de confianza elegido por la víctima); debe ser un adulto en plenas capacidades y que se comprometa a estar en cuerpo presente en todo momento durante las entrevistas o trámites de la denuncia.

\section{Condicionamiento de la denuncia: Fideicomisos y garantías}

La escuela de Derecho de Yale (2008, pp.1-32) tiene un apartado interesante y que podría ser replicado, consistente en un compilado de "Fideicomisos" legales que consisten en "transferir o delegar" bienes, en nuestro caso información a una persona, la cual estará condicionada pero autorizada para el uso de ella en situaciones especiales. Por ejemplo: En la escuela de Derecho de Yale, se permite que una persona haga la denuncia por acoso sexual, pero que se le dé trámite sí y sólo sí otra persona se acerca a denunciar al mismo victimario. Esto con el fin de generar un apoyo a los denunciantes y sumar credibilidad o fuerza a la denuncia.

El anterior objetivo creado por los fideicomisos, también puede generarse mediante el uso de tecnología: la aplicación Callisto Campus (Callisto, 2019), ofrece sus servicios a Universidades y empresas para conectar de manera discreta y segura víctimas de un mismo perpetrador, promoviendo apoyo y cooperación mutua en caso de que decidan denunciar conjuntamente.

Al igual que el Fideicomiso del protocolo de la Universidad de Yale, la aplicación permite la opción de registrar fechas, horas y eventos detallados del acoso y de reportar sí y solo sí otro usuarixs reporta el mismo abusador; lo cual según las cifras de sus desarrolladores aumenta en cinco veces la posibilidad de denunciar, ha propiciado que el 15\% de los usuarios coincidan en su abusador y aumenta en tres veces la rapidez en la denuncia. Dicho reporte puede ser enviado a la institución, a la persona designada para el manejo de las denuncias o a las autoridades competentes según lo disponga cada comprador del servicio.

Saber que no fuiste la única, cambia absolutamente todo.

\section{Conclusiones}

\section{Recomendaciones para un protocolo de la Universidad de Antioquia}

Para concluir, deseo resaltar que la eficacia de los protocolos y rutas de prevención y manejo de denuncias de acoso sexual que se establezcan en cada institución para ambientes académicos o laborales dependerá en una gran medida de cómo sean difundidos en los miembros de cada espacio. No basta con una presentación en power point que explique qué conductas constituyen acoso sexual y las consecuencias para las víctimas y victimarixs. Es imperante que simultáneo a dichos protocolos en las instituciones se promueva una cultura en la que las mujeres sean tratadas como iguales y en la que los miembros de la institución se traten mutuamente con respeto. Que se destinen espacios mensuales a capacitación de todo el personal docente y universitario en temas de género y acoso sexual.

Como lo indica la OIT; Se implica responsabilidad pública y privada: se requiere un marco legal y campañas de información y sensibilización. Promover cambios en la cultura laboral y la instauración de una POLÍTICA EXPRESA CONTRA EL ACOSO SEXUAL, puntual. Debe contar con procedimientos estipulados para 
la denuncia. Y políticas de prevención (2012, p.6).

Es indispensable que las instituciones se comprometan con la creación de rutas, programas de sensibilización y de prevención de acoso sexual, que se hagan cambios no solo de forma, sino de fondo: Acá hay luces en lo que expone Caballero (2003, p. 18).

- Desarrollo de una cultura de rechazo y sanción social contra todas las formas de violencia sexual, ora de incomodidad, coerción o acoso sexual formal.

- El replanteamiento de las concepciones de incomodidad, coerción y acoso sexual formal, puesto que no serían tres fenómenos separados, sino tres formas de violencia sexual.

- Si las formas de acoso sexual formal constituyen expresiones de violencia de género, reflejo de una cultura desigual, se podría proponer el desarrollo de una ética basada en la igualdad entre el varón y la mujer, propendiendo por la superación de la asimetría social

Los protocolos analizados y citados coinciden en su mayoría en asuntos como:

- Políticas de sensibilización en el tema.

- Prohibición EXPLÍCITA de las conductas que pueden ser configuradas como acoso sexual.

- Asegurar entornos saludables y seguros, especialmente para las personas que denuncien.

El "Modelo de atención a las víctimas de Violencia sexual" desarrollado por el Ministerio de Protección social (2011) incluye otra recomendación a mi juicio sumamente valiosa:

- "Promover la ubicación de mujeres en posiciones jerárquicas de poder dentro de la estructura organizacional de los contextos académicos y laborales" (p.86).

Todo ello funciona brindando confianza a posibles denunciantes para hablar con alguien de un rango superior o igual al de su victimarix, y, a su vez, termina siendo una forma de promoción de la paridad en géneros.

El protocolo (Instructivo corto) desarrollado por el ICBF declara que "Es importante que tengas claro que guardar silencio en caso de ser víctima de abuso (incluyen el acoso sexual en su definición) es el PEOR [La mayúscula es mía] error que puedas cometer". (ICBF, 2014, p.4). Se debe resaltar que dicha afirmación puede llegar a ser violenta para la víctima y la puede presionar a tomar decisiones en contra de su voluntad. El lenguaje es importante en estos instructivos, puesto que puede ser crucial para la víctima y quienes los consulten, a la hora de decidir si desean o no denunciar; habría que enfocarse en asegurar que se brindarán todas las garantías de seguridad y no retaliación por parte del victimario como un mejor incentivo.

Otro incentivo menos violento si se quiere apelar a la cooperación de la víctima, pude ser mencionar que su decisión de denunciar puede ser fundamental para impedir que otra persona experimente lo que ella (o él) experimentó.

Rescato del protocolo del ICBF la importancia de la notificación a la víctima de que no está sola, que múltiples entidades y personas están plenamente capacitadas y a su disposición para ayudar en cualquier tema jurídico, médico, psicológico o de otra índole que el haber experimentado dicho abuso sexual (acoso) haya generado.

Incluso la salvedad que se hace al indicar el derecho a la reparación, la asistencia integral y a un trato digno (p.6.) el cual implica:

- Un trato humano (Y digno)

- Que se proteja la intimidad de la víctima

- Que se garantice la protección de la víctima, familiares y testigos de los hechos 
Sobre el personal designado para la recepción de las denuncias se plantea que lo que pude evidenciar es que la mayoría de las universidades recomienda que haya una sola persona encargada de la recepción y manejo de las denuncias; lo cual puede garantizar que dicha tarea sea tratada con celeridad, con experiencia y con un conocimiento profundo de las rutas y protocolos.

Al contrario, el estudio que contempla el protocolo de la Sociedad para la Administración de Recursos Humanos, sugiere que sean varias las personas designadas para dicha tarea en cada estamento, dado que eso representa opciones para que la víctima denuncie con la persona con la que más cómoda se sienta.

Sólo con apoyo formal de las instituciones, puede comenzar una verdadera lucha por abolir prácticas como el acoso sexual.

\section{Referencias bibliográficas}

Aguirre Fernandez, R. (10 de agosto de 2017). Impunidad en violencia contra la mujer es del 96 \% en Colombia. Obtenido de El Colombiano: https://www.elcolombiano.com/ colombia/violencia-contra-la-mujer-en-colombia-EN7078547

Ayres, Ian, "Information Escrows" (2012). Faculty Scholarship Series. Paper 4741.

Caballero, María C., (2003), El acoso sexual en el medio laboral y académico, Facultad de psicología evolutiva, Universidad de Salamanca. Salamanca.

Cárdenas Martínez, E. E., Ramírez Mora, J. M. (2012) El acoso laboral en Colombia: Conceptualización, caracterización y regulación. XX Congreso Mundial de Derecho del Trabajo y Seguridad Social. 1-13.

Castaño Castrillón, J. J., Gonzáles, E. K., Guzmán, J. A., Montoya, J. S., Murillo, J. M., Páez Cala, M. L., Parra, L. M., Salazar, T. V., y Velázquez, Y. (2008) Acoso sexual en la comunidad estudiantil de la Universidad de Manizales. Centro de investigaciones. Pp. 1-17.

Callisto. (2019). Por sobrevivientes, para sobrevivientes. Obtenido de Callisto: https://www. projectcallisto.org/

Consejo Superior Universitario Universidad Nacional de Colombia (16 de Noviembre de 2017). Resolución 1215 de 2017. "Por la cual se establece el Protocolo para la Prevención y Atención de Casos de Violencias Basadas en Género y Violencias Sexuales".

Corporación Sisma Mujer. (2013). Análisis de caso sobre acoso sexual y feminicidio. Recuperado de http://www.sismamujer.org/wp- content/uploads/2016/12/01.2013. An\%C3\%A1 lisis-de-Casos-sobre-Acoso-Sexual-y-Feminicidio-2013.pdf

Cummings, K. M., Madeline, A., Penalties for peer sexual harassment in an Academic Context: The influence of Harasser Gender, Participant Gender, Severity of Harassment, and the presence of ByStanders. (2002). Sex Roles, Vol. 47, Nos. 5/6, September Issue. University of Tampa, Tampa, Florida.

ESADE (s.f) Protocol for prevention and action in situations of mobbing and sexual harassment. Barcelona.

Gaytán Sánchez, P. (2009), Del piropo al desencanto, México D.F, México, Universidad Autónoma Metropolitana.

Instituto Colombiano de Bienestar Familiar-ICBF. (2014). Abuso sexual y Rutas de Atención para víctimas. Guía 7. Recuperado de http://www.icbf.gov.co/portal/page/ portal/.../GuiasDeFamiliaModulo2/M2_Guia7.pdf

Organización Internacional del Trabajo. (2012). Género, salud y seguridad en el trabajo. Recuperado de http://www.ilo.org/wcmsp5/groups/public/---americas/---ro-lima/--sro-san_jose/documents/publication/wcms_227404.pdf 
Oficina Internacional del Trabajo (OIT). (2016). Relaciones Laborales y Negociación Colectiva. Nota de información N4. Recuperado de http://www.ilo.org/global/topics/ collective-bargaining-labour-relations/publications/WCMS_531114/lang--es/index.htm

Project Callisto, (2018), Tech to Combat sexual assault and harassment. Recuperado de https://www.projectcallisto.org/

Ministerio de Educación Nacional. (s.f) Ruta para la atención de situaciones de acoso escolar. Recuperado de: http://www. minieducacion.gov.co

Ministerio de Protección Social (2011) Modelo de Atención Integral en Salud para Víctimas de Violencia Sexual. UNFPA: Colombia

Miller, C. (9 de octubre de 2017) Unintended Consequences of Sexual Harassment Scandals. The New York Times. Recuperado de https://www. nytimes.com/2017/10/09/ upshot/as-sexual-harassment-scandals-spook-men-it-can-backfire-for-women. html

Sapiens Research. (1 de junio de 2019). Ranking Sapiens Research. Obtenido de Sapiens Research: https://www.srg.com.co/usapiens.php

The Anglican Church of Australia-Diocese of Brisbane (2015) Protocol of Dealing with Complains of Sexual Harassment, Sexual Assault or Sexually Inappropriate Behavior.

United States Department of Education. (2001) Revised Sexual Harassment Guidance: Harassment Of Students By School Employees, Other Students, Or Third Parties. (Revised Sexual Harassment Guidance) Recuperado de https://www2.ed.gov/about/ offices/list/ocr/docs/shguide.html

Universidad de Antioquia. (13 de septiembre de 2005). Acuerdo 297 Universidad de Antioquia. Obtenido de Universidad de Antioquia: http://avido.udea.edu.co/autoevaluacion/documentos/institucional/regimen_disciplinario_personal_docente_acdo_ sup_297_2005.pdf

Universidad de Antioquia. (mayo de 2006). El desarrollo de la Universidad de Antioquia 1995 - 2006. Obtenido de Rectoría oficina de Planeación: EL DESARROLLO DE LA UNIVERSIDAD DE ANTIOQUIA 1995 - 2006

Universidad de Antioquia. (2018). Universidad de Antioquia plataforma de cosulta. Obtenido de Universidad de Antioquia: https://normativa.udea.edu.co/Documentos/ Consultar

Universidad de Antioquia. (marzo de 2019). Informe de gestión 2018. Obtenido de Universidad de Antioquia: http://www.udea.edu.co/wps/wcm/connect/udea/ed738d52b883-40f1-9594-978149ff05f6/informe-gestion-2018.pdf?MOD=AJPERES\&CVI$\mathrm{D}=\mathrm{mDzA} 2-\mathrm{u}$

Universidad Nacional de Colombia. (2017). Resolución 1215 de 2017 (Del 16 de noviembre). Por la cual se establece el protocolo para la Prevención y Atención de casos de Violencias basadas en Género y Violencias Sexuales. Recuperado de http:// www.legal.unal.edu.co/rlunal/home/doc.jsp?d_i=89782

University of Toronto (2004) SEXUAL HARASSMENT PROTOCOL: Sexual Harassment Complaints involving Faculty and Students of the University of Toronto arising in University-Affiliated Health Institutions.

Valles, M. (1999). Técnicas cualitativas de la investigación social. Reflexión metodológica y práctica. España: Editorial Síntesis.

Yale University. (2018). Preventing and Responding to Sexual Misconduct: Building a Climate of safety and Respect at Yale. Recuperado de https://smr.yale.edu/sites/default/files/files/Guide-Preventing-and-Responding-to-Sexual-Misconduct.pdf 